\title{
Innsbruck cantat
}

\section{Ein Pilotprojekt zur Zusammenarbeit zwischen ausgewählten Tiroler Volksschulen und der Musikschule der Stadt Innsbruck}

\section{Elisabeth Nagiller-Rendl ${ }^{1}$}

https://doi.org/10.53349/resource.2021.i16.a987

\section{Zusammenfassung}

„Innsbruck cantat" stellt die erste Evaluation des Kooperationsprojekts „Singende Schule“ dar. In diesem Pilotprojekt kooperieren verschiedene öffentliche Innsbrucker Volksschulen mit der Musikschule der Stadt Innsbruck. Die empirischen Untersuchungen erfolgten in einem quantitativ-qualitativen Setting mittels Fragebogen für die Schüler*innen mit großteils geschlossenen Fragestellungen und Leitfadeninterviews mit den Projektverantwortlichen bzw. dem Schulpersonal zum musikalischen und sozialen Mehrwert dieser Kooperation. Im Rahmen des Regelunterrichts übernehmen Musikpädagog*innen Teile des Unterrichtsfaches Musikerziehung in Projektklassen. Die Klassenlehrer*innen sind während des Unterrichts anwesend, beobachten und unterstützen die Arbeit der Musikpädagog*innen. Für die Evaluation wurden zwei Volksschulen ausgewählt, an denen die Autorin seit Jahren als Musikpädagogin im Projekt im Einsatz ist. Ziel der Untersuchung war die detaillierte Identifizierung des Mehrwerts des Projekts. Gleichzeitig galt es, Schwachstellen im Projekt zu erkennen sowie identifizierte Qualitätsverbesserungsmöglichkeiten aufzuzeigen. Nach Auswertung aller Daten stellen die Ergebnisse dem Projekt ein positives Zeugnis aus. So sollte das Projekt "Singende Schule“ in Zukunft unbedingt weitergeführt werden. Die differenzierte Betrachtung einzelner Teilergebnisse wird im Beitrag diskutiert.

\begin{tabular}{ll} 
Schlüsselwörter: & Keywords: \\
Singende Schule & singing school \\
Kooperationsprojekt & cooperation project \\
Musikschule & music school \\
Musikpädagogik & music education \\
\hline
\end{tabular}

\section{Abstract}

"Innsbruck cantat" is the first evaluation of the cooperation project "Singing School". In this pilot project different public elementary schools work together with Innsbruck's music school.

Music school teacher various parts of the music lessons in certain project classes throughout the whole school year. The class teachers are also present during these lessons and observe and support the work of the music school educators. The author is part of this project and two elementary schools where she is working as a music school educator for several years have been chosen for the evaluation.

The aim of the investigation was to find out the added value of the project in detail. On the other hand it was necessary to identify weak points and to show quality improvement opportunities. After evaluation of all data it can be resumed that the project was successful. "Singing School" should definitely be continued. More detailed results will be discussed in the article.

\section{Einleitung}

Seit einigen Jahren gibt es vermehrt institutionalisierte Kooperationen von Musikschulen mit allgemeinbildenden Schulen. In Tirol betrifft das vor allem die Landeshauptstadt Innsbruck.

\footnotetext{
${ }^{1}$ Kirchliche Pädagogische Hochschule Edith Stein, Stiftshof 1, 6422 Stams.

Korrespondierende Autorin. E-Mail: elisabeth.nagiller@gmail.com
} 
Hierbei arbeitet die städtische Musikschule seit 2015 im Projekt „Singende Schule“ mit Volksschulen zusammen. Derzeit sind zehn Innsbrucker Volksschulen in das Projekt involviert. Beim Projekt „Singende Schule“ gestalten Musikpädagog*innen den Unterricht Musikerziehung im Rahmen des Regelunterrichts der Pflichtschule. Dies erfolgt ohne explizite Anmeldung der Kinder durch die Erziehungsberechtigten. Damit kommen alle Kinder einer Klasse in den Genuss des musikalischen Zusatzangebots.

Von Pädagog*innen und Eltern wird das Projekt sowohl in der Wirkung nach innen als auch nach außen immer wieder positiv bewertet. Das zeigt sich vor allem dann, wenn musikalische Darbietungen als Ergebnisse des Unterrichts im Rahmen von Konzerten, Feiern und Aufführungen öffentlich gezeigt werden.

In diesem Beitrag wird zunächst das Projekt vorgestellt und anschließend werden Untersuchungsergebnisse, die mittels Schüler*innenfragebögen und qualitativer Leitfadeninterviews erhoben wurden, dargestellt. Die Fragebögen wurden im Schwerpunkt mittels deskriptiver Statistik ausgewertet. Die Interviews wurden mittels üblicher qualitativer Methoden (Kategorienbildung) analysiert. In einem dritten Schritt wurden die Einzelergebnisse trianguliert.

Eine Literaturrecherche in diesem Zusammenhang ergab, dass empirische Untersuchungen oder Belegstudien zum gegenständlichen Projekt nicht vorliegen. Es war deshalb naheliegend, „Innsbruck singt“ einer Evaluation zu unterziehen. Die Auswertung der Ergebnisse kann und soll auch eine zukünftige Optimierung der institutionellen Zusammenarbeit und der inhaltlichen Konzeption der Unterrichtseinheiten nach sich ziehen. Die vorliegenden Ergebnisse sollten im Sinne einer Pilotstudie einen Ausgangspunkt für weitere Untersuchungen darstellen.

\section{Beschreibung des Projektes „Singende Schule“}

In der „Singenden Schule“ sollen möglichst viele Bereiche der musikalischen Erziehung von Kindern berücksichtigt werden, wiewohl der Schwerpunkt auf dem Singen liegt. In der Broschüre „Kooperationen von Schulen und Musikschulen“, die von der Arbeitsgruppe "Musikschulen - Schulen“ im Rahmen der "Steuergruppe Musik \& Bildung" 2013 erstellt wurde, werden sechs verschiedene Modelle der Kooperation beschrieben. Das Projekt "Singende Schule“ findet im Modell C „Teamteaching mit Musikschullehrkraft“ die Voraussetzungen und Rahmenbedingungen für diese Art der Kooperation (vgl. BM:UKK, (2013), S. 8). Bewegung, Rhythmik, Body-Percussion, Umgang mit Instrumenten, Gehörbildungs- und Sensibilisierungsübungen, elementare Musiklehre können und sollen dabei in die aktive Vokalarbeit integriert werden. Der Hauptteil der Unterrichtsstunde besteht aus Stimmbildungsübungen und der Vermittlung eines vielfältigen Liedgutes, hauptsächlich in deutscher Sprache. Die vorwiegend auswendige Erarbeitung der Liedtexte zielt in Richtung Förderung der Konzentration und Merkfähigkeit der Kinder.

Die gesungene Literatur wird grundsätzlich vom Singschullehrkollegium bzw. von dem*der Singschulleiter*in vorbereitet. Selbstverständlich können auch vonseiten der Volksschullehrkraft Lieder vorgeschlagen und in den Unterricht eingebaut werden.

Eine Unterrichtsstunde in der "Singenden Schule“ hat nicht nur die Vermittlung eines neuen Liedes oder die Wiederholung bereits erarbeiteter Lieder zum Inhalt. Es geht - wie oben erwähnt - um eine am Lied orientierte und mit mehreren Bereichen der Musikerziehung vernetzte Vokaldidaktik. Eine vielfältige und möglichst wenig monotone Arbeitsweise ist hier notwendig. Nähere Details zum Projekt „Singende Schule“ sind in der Festschrift „200 Jahre Musikverein, Musikschule, Konservatorium in Innsbruck“ (2018) bzw. im Leitfaden der Musikschule der Stadt Innsbruck (2016) nachzulesen, weil sie den Umfang dieses Artikels sprengen würden.

\subsection{Mitwirkung der Volksschullehrperson}

Die Volksschullehrer*innen können sich in verschiedenster Art und Weise in den Unterricht einbringen. Zum Beispiel können sie bei der Auswahl der Lieder mitentscheiden oder ein Hirtenspiel, Musical etc. vorschlagen. Darüber hinaus ermöglicht die Zusammenarbeit der Lehrkräfte eine Aufteilung der Lehrtätigkeit (eine Lehrperson übernimmt zum Beispiel die Klavierbegleitung, die andere das Dirigat). Ein Thema ist u. a. die Sonderbetreuung von kleineren Gruppen, um besser auf die Bedürfnisse der Kinder eingehen zu können. 
Im Regelunterricht sollten jedenfalls die erlernten Lieder vertieft werden, um die Musikschulpädagog*innen aus der Musikschule zu unterstützen und somit den Unterrichtsfortschritt für die nächste(n) Musikstunde(n) zu gewährleisten. Insofern ist eine enge Zusammenarbeit und Absprache zwischen den Pädagog*innen notwendig (vgl. Leitfaden der Musikschule der Stadt Innsbruck, 2016, S. 2f.).

Grundsätzlich soll das Projekt „Singende Schule“ nicht in isolierten Musikstunden betrachtet werden. Vorrangig ist die Zielperspektive einer Förderung der musikalischen Anlagen von Kindern im Grundschulalter mit Schwerpunkt „Singen“. Durch den Einsatz der Musikschulpädagog*innen kann vor allem die Singstimme der Kinder professionell gefördert werden. Dabei geht es in erster Linie um Atmung, Stimmsitz, Artikulation und Intonation. Gerade ein intensiver Musikunterricht, der im vokalpädagogischen Bereich von einer ausgebildeten Fachkraft geleitet wird, kann bei schulinternen Feierlichkeiten eine willkommene Bereicherung sein. Andererseits sollten die Kinder aus den jeweiligen Singklassen der Volksschule auch bei Konzerten der Musikschule mitwirken und so Kontakt zur möglichen zukünftigen musikalischen Ausbildungsinstitution knüpfen können.

Ein Idealzustand ist dann gegeben, wenn beide Institutionen in diesem Projekt eng zusammenarbeiten.

\subsection{Auswirkungen bzw. Veränderungen für Lehrpersonen der Musikschule}

Für den*die Musikschullehrer*in ist die Einführung der „Singenden Schule“ in mehrfacher Hinsicht eine große Herausforderung. Da der Unterricht im Rahmen des Regelunterrichts der Pflichtschule stattfindet, trifft die Lehrkraft der Musikschule nun auf Kinder, die sich nicht grundsätzlich freiwillig zur Singstunde bzw. Singschule angemeldet haben. Alle Kinder einer Klasse sind beteiligt, auch jene, die wenig oder keine Affinität zur Musik haben bzw. von ihren Eltern diesbezüglich nicht bewusst gefördert werden. Alle Kinder zu motivieren und sie als mitunter inhomogene Gruppe methodisch und didaktisch entsprechend zu betreuen, bedeutet für die Lehrperson, ein hohes Maß an Geschick, Können und auch Energie aufbringen zu müssen.

Vor allem bei Veranstaltungen (Konzerte, öffentliche Auftritte), die außerhalb des Regelunterrichts stattfinden, kann es zum Problem werden, wenn im Elternhaus nicht genügend Überzeugungsarbeit geleistet wird und die Lehrperson aus der Musikschule mit wenig helfender Motivation von zu Hause und auch mit Absenzen der Kinder rechnen muss.

Das Abhalten von Unterrichtssettings in der Gruppengröße einer Klasse stellt die*den Musikpädagogin*Musikpädagogen vor besondere Herausforderungen. Eine für die Lehrperson der Musikschule meist unbekannte Problematik ist das Halten der Disziplin in der Klasse. Im Einzel- bzw. Ensembleunterricht der Musikschule stellt das in der Regel kein Problem dar. In einer Klassensituation kann Disziplin aber eine Schlüsselrolle für das Gelingen von Unterricht einnehmen.

Im Umgang mit Klassen gibt es noch weitere Faktoren, die für die Lehrenden aus dem Musikschulbereich Neuland bedeuten. Die Heterogenität der Schüler*innen innerhalb einer Klasse ist enorm. Allen Kindern die bestmögliche Förderung zukommen zu lassen, ist keine leichte Aufgabe. Auch schulorganisatorische Rahmenbedingungen stellen Anforderungen an die Lehrperson, die in einer Musikschule nicht relevant sind. Hier sind unter anderem die Aufsichtspflicht in den Pausen oder der Wechsel der Kinder von einer Klasse in den Musiksaal bzw. Musikraum zu nennen. Weiters fällt auf, dass im städtischen Bereich eher eine gesellschaftlich „gehobene Schicht“ die Musikschule besucht. Auch der Anteil von Schüler*innen mit Migrationshintergrund ist an der Musikschule Innsbruck sehr gering. Das entspricht im Allgemeinen nicht der Regelpopulation einer städtischen Volksschulklasse.

Ein weiterer Aspekt ist die stimmliche Anforderung an die Singlehrer*innen. Das Singen und die vokale Arbeit generell nehmen ca. 75 \% der Unterrichtseinheit ein. Es gibt selten „Ruhephasen“ für die Stimme der Lehrperson. Wenn nun bis zu vier Unterrichtseinheiten am Vormittag im Projekt „Singende Schule“ gehalten werden und zumeist nachmittags der Unterricht an der Musikschule fortgesetzt wird, ergeben sich mitunter Arbeitstage von 8.00 Uhr bis 19.00 Uhr. Diese zehren vor allem sehr an der stimmlichen Substanz. Dabei geht es nicht nur um den „bloßen Einsatz" der Stimme. Gerade innerhalb der Gesangspädagogik sind die stimmliche Vorbildwirkung und der stimmliche Ausdruck beim Unterrichten von großer Bedeutung, wie es auch Kurtz betont: „Eine gesunde, tragfähige, klangschöne, ökonomisch eingesetzte Stimme und eine ausdruckstarke, modulationsfähige Sprechweise sind im Klassenzimmer von unschätzbarem Wert“" (Kurtz, 2018, S. 9).

Positiv ist zu erwähnen, dass der Klassenunterricht für das Lehrer*innenkollegium der Musikschule neue Unterrichtsformen und Chancen ermöglicht, die im Einzelunterricht nicht praktiziert bzw. wahrgenommen werden 
können. Verschiedene Sozialformen, wie zum Beispiel der Unterrichtskreis, bieten hier der Lehrperson die Gelegenheit, mitten unter den Schüler*innen zu agieren und an gruppendynamischen Prozessen teilzuhaben, die sich so im Musikschulunterricht zumeist nicht ergeben können.

Diese Ausführungen beruhen auf persönlichen Erfahrungen, die im Rahmen einer wissenschaftlich fundierten Folgeuntersuchung zu erheben wären.

\subsection{Vergleich der Ausbildung von Volksschul- und Musikschulpädagog*innen}

Primarpädagog*innen sind „Generalisten“, die mehrere Fächer unterrichten. Musikerziehung hat im Laufe des Studiums im Allgemeinen keine größere Bedeutung als alle anderen Fachgegenstände, wie das Curriculum für das Lehramt Primarstufe der Pädagogischen Hochschule Tirol zeigt (PHT, 2021, Zugriff am 1.7.2021 unter http://ph-tirol.ac.at/node/90).

Musikschulpädagog*innen erhalten am Konservatorium oder an einer Musikuniversität eine hochqualifizierte musikalische Ausbildung in ihrem jeweiligen Fach (instrumental oder vokal). Es findet keinerlei pädagogische Schulung für den Umgang mit Volksschulklassen statt Die didaktisch-methodische Ausbildung beschränkt und bezieht sich ausschließlich auf den musikalischen Einzel-, Gruppen- und Ensembleunterricht (Tiroler Landeskonservatorium, 2021 Zugriff am 1.7.2021 unter https://www.konstirol.at/studien-und-curricula/igp-ba/). Gesangspädagog*innen erhalten also eine spezialisierte Fachausbildung im Vokalbereich. Lehrpersonen im Primarbereich durchlaufen eine sehr breite didaktisch-methodische Ausbildung, der künstlerische und fachwissenschaftliche Anteil ist hier im Vergleich geringer.

\section{Untersuchungsdesgin zum Projekt}

Im Sinne einer Evaluationsstudie wurde das im Folgenden beschriebene Untersuchungsdesign aufgebaut.

\subsection{Forschungsfeld und Forschungsfrage}

Das zu untersuchende Forschungsfeld ergibt sich aus dem Anspruch der Studie, das Projekt „Singende Schule“ an zwei ausgewählten Innsbrucker Schulen im Detail darzustellen und zu evaluieren.

Folgende zentrale Forschungsfrage wurde hierbei in den Fokus gestellt: „Bringt die Zusammenarbeit zwischen Volksschule und Musikschule einen musikalischen und sozialen Mehrwert für Schüler*innen bzw. für die beteiligten Lehrpersonen?"

In einem ersten Schritt wurden die zu befragenden Personengruppen festgelegt. Es wurde entschieden, dass sowohl 251 Schüler*innen als auch die zwei Schulleiterinnen der Schulen sowie drei Lehrpersonen befragt werden. Zusätzlich erschien es wichtig, auch die Sichtweise des Leiters der Musikschule Innsbruck sowie jene des Singschulleiters in Erfahrung zu bringen.

Für die vorliegende Studie ist zu vermerken, dass die von den Schüler*innen erhobenen Daten nahezu eine Grundgesamtheit an den beiden Schulen darstellen (85\% der Schüler*innen wurden befragt).

Zu beachten ist, dass die Ergebnisse zwar eine hohe Gültigkeit für beide Schulen haben, aber für das gesamte Projekt „Singende Schule“ nur eingeschränkt herangezogen werden dürfen. Die Ergebnisse haben für das Gesamtprojekt jedoch insofern Relevanz, weil sie eine gewisse Tendenz abbilden. Für allgemein gültige Aussagen über das Projekt wäre deshalb eine Gesamtevaluation an allen zehn Volksschulen erforderlich. 


\subsection{Untersuchungsinstrumente}

Es wurden sowohl quantitativ empirische als auch qualitativ empirische Erhebungsinstrumente erarbeitet und eingesetzt. Schlussendlich fiel die Entscheidung, für die Befragung der Schüler*innen einen Fragebogen mit durchgehend geschlossenen Fragestellungen zu entwickeln. Für die zu befragenden Lehrpersonen und Schulleiterinnen sowie für den zu befragenden Singschulleiter und den Musikschuldirektor erschien die Methode des Leitfadeninterviews adäquat.

\subsection{Quantitative Herangehensweise}

Der Fragebogen für die erste Schulstufe enthält insgesamt zehn Fragestellungen. Neben soziodemographischen Fragen werden vor allem Fragen zur Rezeption des Projekts gestellt. Unterschiedliche positive bzw. negative Polungen ermöglichen eine Plausibilitätsprüfung der Antworten. Die Fragen vier, sechs und zehn beinhalten jeweils mehrere Antwortmöglichkeiten, bei denen die Schüler*innen das für sie Zutreffende ankreuzen sollten.

Der Fragebogen für die Schüler*innen der 2.-4. Schulstufen ist etwas ausführlicher gehalten. Er enthält insgesamt vierzehn Fragen bzw. Fragenkomplexe. Inhaltlich ist er ansonsten dem Fragebogen für die ersten Schulstufen sehr ähnlich, dies vor allem deshalb, um eine Vergleichbarkeit sicherzustellen.

\section{Durchführung der Fragebogenuntersuchung in den Klassen}

Nachdem die zu befragende Gruppe von Schüler*innen zwischen sechs und maximal zwölf Jahre alt war, wurde entschieden, dass die Autorin die Befragung im Rahmen einer Stunde selbst durchführt. Damit schien gewährleistet, dass das Setting möglichst immer gleich ablief und dass auftauchende Fragen und Unklarheiten zum Fragebogen auf gleiche Weise beantwortet werden konnten. Die Beantwortung der Fragebögen beanspruchte pro Klasse ca. 20-25 Minuten.

Neben vielen positiv gepolten Fragen wurden ganz gezielt die folgenden Fragestellungen aufgenommen:

- Das Singen macht mich traurig.

- Durch die Singstunden gibt es mehr Streit in unserer Klasse.

- Musik interessiert mich überhaupt nicht.

Die beiden ersten Fragen stellen Schlüsselfragen in Bezug auf die emotionale Befindlichkeit der Kinder und das soziale Gefüge der Klasse dar. Eine hohe Zustimmung zu diesen Fragestellungen würde ein Alarmsignal für das Projekt darstellen.

Die dritte Frage schätzt zum Ersten die allgemeine Einstellung der Schüler*innen zur Musik ein. Das ermöglicht eine gute Vergleichbarkeit mit anderen Fragestellungen. Zum Zweiten ist bei einem hohen Grad der Zustimmung zu erwarten, dass das Projekt „Singende Schule“ und dessen Inhalte eher weniger Anklang bei den Volksschulkindern finden wird.

Da die Anführung aller Fragen den Umfang des Artikels sprengen würde, werden an dieser Stelle die Einzelfragen in inhaltlich zusammengehörige Fragenblöcke zusammengefasst, vorgestellt und diskutiert.

Einleitend wird das Alter und Geschlecht anonymisiert abgefragt, um vergleichende statistische Auswertungen zu ermöglichen.

\begin{tabular}{|l|l|l|}
\hline \multicolumn{1}{|c|}{ Fragenblock } & \multicolumn{1}{|c|}{ Inhalt } & \multicolumn{1}{c|}{ Erläuterung } \\
\hline Fragenblock 1 & $\begin{array}{l}\text { Häusliche Beschäftigung } \\
\text { mit Musik }\end{array}$ & $\begin{array}{l}\text { Der erste Fragenblock erörtert, wie sich Kinder zu Hause } \\
\text { mit Musik beschäftigen, und dient vor allem dazu, dass au- } \\
\text { ßerschulische Umfeld der Kinder näher zu betrachten. Ab- } \\
\text { gefragt werden die Häufigkeit der Beschäftigung mit Musik } \\
\text { zuhause, die Inhalte der Beschäftigung (singen, Musik hö- } \\
\end{array}$ \\
& $\begin{array}{l}\text { ren, tanzen, ein Instrument spielen), ob ein Instrument ge- } \\
\text { spielt wird und wo der Unterricht erfolgt. }\end{array}$ \\
\hline
\end{tabular}




\begin{tabular}{|l|l|l|}
\hline Fragenblock 2 & $\begin{array}{l}\text { Musikunterricht in der } \\
\text { Schule }\end{array}$ & $\begin{array}{l}\text { Einleitend wird in diesem Fragenblock erhoben, wie oft Mu- } \\
\text { siksequenzen während einer Woche in der Klasse stattfin- } \\
\text { den. Im Folgenden wird die Einstellung der Kinder zum Mu- } \\
\text { sikunterricht erfragt. Ganz gezielt wird hier auch die emoti- } \\
\text { onale Komponente angesprochen. Wie weit „berührt" der } \\
\text { Unterricht die Kinder? } \\
\text { Im Detail werden die Vorlieben der Kinder für bestimmte } \\
\text { Inhalte des Musikunterrichts durch Multiple-Choice Fragen } \\
\text { (Die Musikstunde gefällt mir, weil ...) abgefragt. }\end{array}$ \\
\hline Fragenblock 3 & $\begin{array}{l}\text { Musikunterricht im Ver- } \\
\text { gleich zu anderen Fä- } \\
\text { chern }\end{array}$ & $\begin{array}{l}\text { Fragenblock 3 vergleicht in erster Linie die Beliebtheit des } \\
\text { Musikunterrichts mit jener anderer Fächer und soll die } \\
\text { Gründe dafür an die Oberfläche führen. } \\
\text { Abgefragt werden auch die Einstellung und die Motivation } \\
\text { der Kinder zur Schule insgesamt. } \\
\text { Den Schluss bildet die Frage, wie oft sich die Kinder Musik } \\
\text { in der Schule überhaupt wünschen. }\end{array}$ \\
\hline Fragenblock 4 & $\begin{array}{l}\text { Sozial-emotionale Aus- } \\
\text { wirkungen des Musikun- } \\
\text { terrichts auf Kinder }\end{array}$ & $\begin{array}{l}\text { Im abschließenden Fragenblock werden die sozial-emotio- } \\
\text { nalen Auswirkungen des Musikunterrichts auf Kinder bzw. } \\
\text { die Gruppe/Klasse, die die Kinder selbst wahrnehmen, er- } \\
\text { hoben. }\end{array}$ \\
\hline
\end{tabular}

\subsection{Qualitative Herangehensweise mittels Leitfadeninterviews}

Das Leitfadeninterview als eine Form des problemzentrierten Interviews ermöglicht es den Interviewenden, bestimmte Fragen zum jeweilig besten Zeitpunkt während des Interviews zu stellen (vgl. Diekmann, 2010, S. 542), was sich gerade im Hinblick auf die zu behandelnde Thematik als sinnvoll erwies.

Für die vorliegende Arbeit wurde zunächst ein zentraler Leitfaden entwickelt. Dies geschah vor allem deshalb, um eine Vergleichbarkeit zwischen den einzelnen befragten Personengruppen zu gewährleisten.

Dieser wurde von mehreren Fachleuten im Sinne von „Critical Friends" einer kritischen Analyse unterzogen. Aus dem adaptierten zentralen Leitfaden wurden vier inhaltlich leicht variierende Leitfäden bezogen auf die im Feld tätigen Personengruppen entworfen (Direktor der Musikschule, Leiter der Singschule, Schulleiterinnen, Lehrpersonen).

Jedes Interview wurde zunächst als Audiodatei aufgezeichnet und anschließend transkribiert. Die Auswertung erfolgte mittels qualitativer Inhaltsanalyse.

\section{Darstellung der Ergebnisse}

Die Auswertung der Schüler*innenfragebögen erfolgte EDV-gestützt. Zur Anwendung kamen vor allem Methoden der deskriptiven Statistik. Besonders interessant erschien der Vergleich zwischen Buben und Mädchen. Deshalb erfolgte auch eine spezielle Auswertung in dieser Hinsicht.

Da die Daten bei den allermeisten Fragen nur das Niveau einer Ordinalskala erreichten, erschien als einzige Möglichkeit der Prüfung die Durchführung eines Chi²-Tests (vgl. Brosius, 2004, S. 422). 


\subsection{Zusammenschau der Ergebnisse des Fragebogens der Schüler*innen}

Im Zuge der Untersuchung wurden 251 Schüler*innen sowie zwei Experten der Musikschule und fünf Expertinnen der Volksschule zum Projekt „Singende Schule“ befragt.

Nachstehend werden die wichtigsten Ergebnisse nach Auswertung des Schüler*innenfragebogens dargestellt.

Eine der ersten Fragen bezog sich auf die häusliche Beschäftigung mit Musik, weil daraus möglicherweise angenommen werden kann, dass Schüler*innen, die sich zuhause mit Musik beschäftigen, aufgeschlossener gegenüber dem Musikunterricht sind.

Das häusliche Musikhören stellt, bezogen auf die gestellten Fragen, bei allen Kindern die absolute Lieblingsbeschäftigung dar, gefolgt vom Singen.

Musikhören sollte daher als Teilbereich in den Musikunterricht immer wieder einbezogen werden. Dabei wäre das konkrete Eingehen auf jene Musik, die zuhause gehört wird, im Unterricht möglich. Im Unterricht selbst wird diese Musik besprochen und aktiv verwendet. Sehr gut möglich wäre das in Bewegungssequenzen oder auch in Hörsequenzen, in denen man sich kreativ (z. B. Malen zur Musik) beschäftigt. Zudem könnte die zuhause gehörte Musik auch einen Einstieg für andere Inhalte in den Projektstunden bilden.

Da die Schüler*innen den Ergebnissen zufolge zuhause häufig singen und das auch gerne tun, sollte einerseits das Singen weiterhin den Schwerpunkt im Projekt darstellen und andererseits kann daran im weiteren Schulunterricht gut und einfach angeknüpft werden, um so bisher weniger beachtete oder vernachlässigte Kompetenzbereiche wie Tanzen, Bewegen und Darstellen in Verbindung mit Musik zu fördern.

Eine weitere wesentliche Fragestellung zur Beantwortung der übergeordneten Forschungsfrage war die allgemeine Stundenbewertung der Musikstunde, die jeweils von der externen Musikpädagogin gehalten wird. Hier gaben rund 87 \% der Erstklässler und 90\% der Schüler*innen der 2.-4. Klassen an, dass sie diesen Unterricht als "ganz toll“ bzw. „toll“ bezeichnen würden.

Von zentraler Bedeutung für die Weiterentwicklung des Projekts war auch die Frage, welche musikalischen Inhalte von Seiten der Schüler*innen besonders anerkannt werden, da mit einem gut überlegten, differenzierten und auch auf die Bedürfnisse der Kinder abgestimmten Einsatz der Inhalte die Motivation im Musikunterricht erhöht werden kann.

Im laufenden Projekt haben die Kinder in Summe große Freude am Musikunterricht. Auch die Lieder scheinen großteils gut ausgewählt, weil sie bei den Schüler*innen sehr gut ankommen. Dabei wurden bei der Auswahl der Lieder vor allem die folgenden Aspekte beachtet:

* Lieder in deutscher Sprache

* Vermittlung möglichst unterschiedlicher Musikrichtungen (Jazz, Pop, Rock, ...)

* Volkslieder aus dem alpenländischen Raum (Vermittlung der heimischen Kultur)

Nachstehende Grafik zeigt den Vergleich zwischen der 1. Schulstufe und der 2.-4. Schulstufe: 


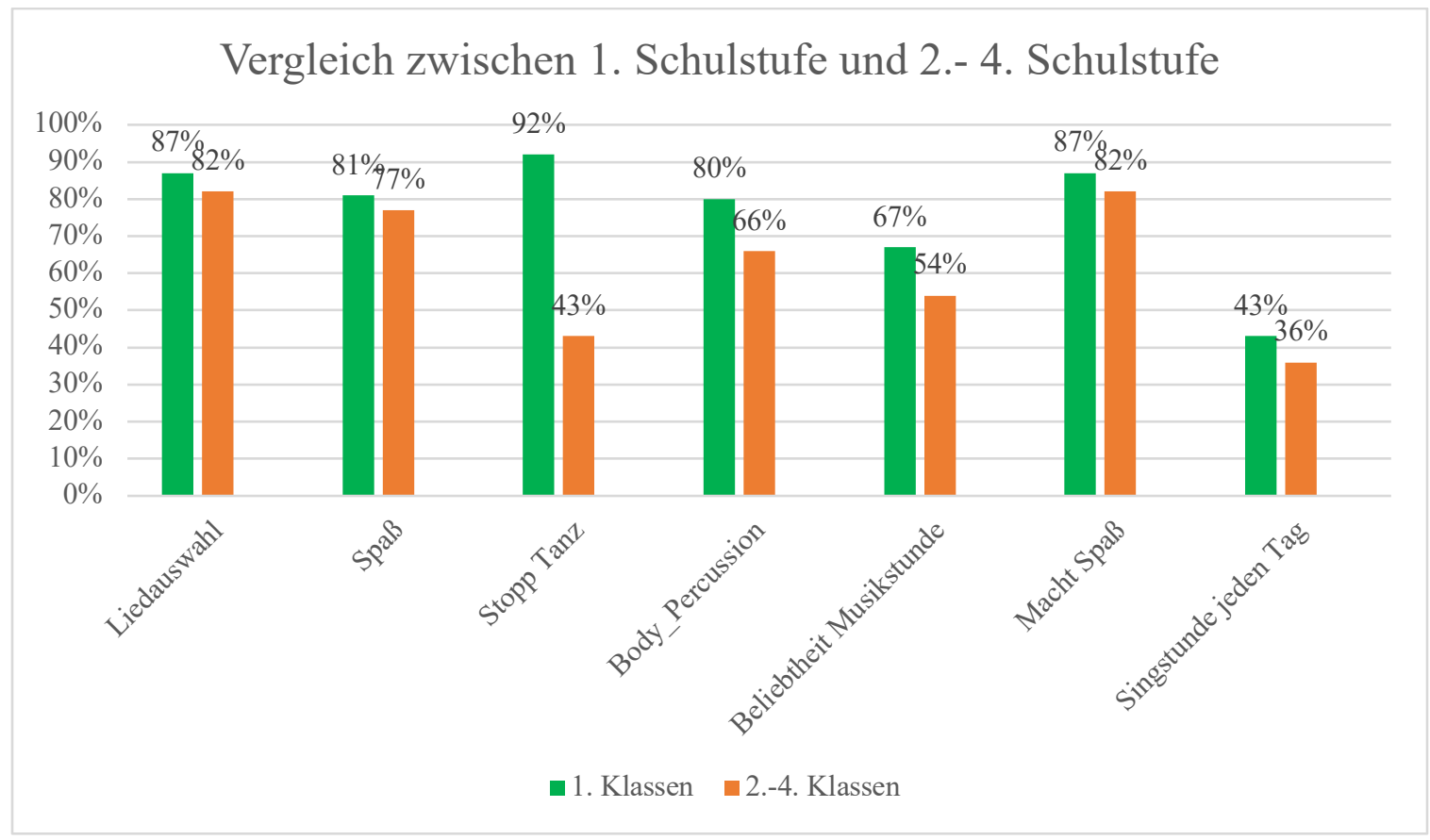

Zusammenfassend kann der Vergleich der Schulstufen auf folgenden Nenner gebracht werden:

Das Antwortverhalten der befragten Volksschüler*innen fällt sehr ähnlich aus. Auffallende Unterschiede zeigen sich in den Kategorien „Bewegung", „Body-Percussion“ und "Beliebtheit der Musikstunde“.

Erwähnenswert ist, dass beide Gruppen bei der Bewertung der Bewegung zur Musik weit auseinanderliegen. Während dieser Zugang in den 1. Klassen von $92 \%$ gerne angenommen wird, stimmten hier lediglich $43 \%$ der Kinder aus den 2.-4. Klassen zu.

Dieses Ergebnis sollte in die zukünftige Vorbereitung der Unterrichtsstunde einfließen, indem vor allem dem Bewegungsdrang der 6- bis 7-Jährigen Rechnung getragen wird.

Die Zustimmung zum Projekt "Singende Schule“ ist bei den 1. Klassen bei allen Fragestellungen höher als jene der befragten Kinder der 2.-4. Klassen.

Ein Punkt, warum die jüngeren Kinder dem Projekt noch mehr Begeisterung entgegenbringen, scheint zu sein, dass die "Offenohrigkeit" ca. bis zum 8. Lebensjahr anhält. Unter diesem Begriff versteht man, dass Kinder offen gegenüber jeglichen Musikrichtungen sind. Jedem*r Lehrer*in sollte bewusst sein, dass diese mit zunehmendem Alter stetig abnimmt. Diese These stützt sich auf die Untersuchungen von Gembris und Schellberg, die die musikalischen Interessen von Kindern im Grundschulalter untersuchten und dabei feststellten, dass bei jüngeren Kindern (bis zum zweiten Grundschuljahr) die Offenohrigkeit größer ist als bei den Älteren (vgl. Auhagen et al., 2007, S. 71). „In musikpädagogischer Hinsicht kann man sagen, dass im Allgemeinen die Chancen, Schülerinnen und Schüler mit einem breiten Spektrum an musikalischen Stilen vertraut zu machen, umso günstiger sind, je früher man damit anfängt" (Auhagen et al., 2007, S. 90).

Schlussfolgernd könnte bei der Auswahl der Inhalte noch mehr darauf geachtet werden, welche Wünsche die Kinder haben. Es wäre zu überlegen, Bewegungssequenzen noch besser gemeinsam abzustimmen bzw. bei geplanten größeren Projekten die Kinder mitentscheiden zu lassen, was konkret umgesetzt werden soll.

Folgend noch einige wichtige Ergebnisse:

Wichtig scheint den Schüler*innen auch die Teilnahme an öffentlichen Auftritten zu sein. Die Schüler*innen wurden befragt, ob sie musikalische Auftritte toll finden. $63 \%$ aller Altersstufen kreuzten bei der Befragung diesen Satz an. Auch im österreichischen Lehrplan der Volksschule steht im Gesetzestext in Bezug auf eine musische Bildung: Schüler*innen sollen dazu „,befähigt werden, am Wirtschafts- und Kulturleben Österreichs, Europas und der Welt Anteil zu nehmen ..." (Wolf, 2018, S. 15). 
Dieses Ergebnis sollte bereits in der Jahresplanung berücksichtigt werden, damit wirklich alle Kinder im Laufe eines Jahres in den Genuss eines öffentlichen Auftrittes kommen. Bei der Weiterentwicklung des Projekts sollte die Teilnahme an öffentlichen Veranstaltungen noch mehr forciert werden.

Eine Fragestellung zielte auf den Vergleich mit anderen Unterrichtsstunden in der Volksschule ab. Die Schüler*innenantworten ergaben hier für die musikalischen Projektstunden eine hohe Präferenz. Dies spricht ebenfalls für die Beibehaltung und Fortführung der "Singenden Schule“.

Die Befragungsergebnisse aus den beiden untersuchten Volksschulen stellen dem Projekt im Gesamten ein hervorragendes Zeugnis aus.

Die Schüler*innen gehen allgemein sehr gerne in die Schule, besonders schätzen sie auch das Projekt. Somit sind aufgrund dieser Ergebnisse grundlegende Änderungen in Bezug auf die Konzeption der "Singenden Schule“ nicht notwendig.

Weiter wurden die Schüler*innen bei der Untersuchung auch zu ihrem emotionalen Wohlbefinden befragt. $80 \%$ der Volksschulkinder gaben der Aussage "Miteinander singen macht Spaß" ihre Zustimmung. Somit erhielt das Singprojekt auch bei diesem Aspekt eine hohe Anerkennung, was ebenfalls als Begründung für die Sinnhaftigkeit des Projekts gedeutet werden kann.

Abschließend können die Ergebnisse der Befragung der Schüler*innen in zwei Sätzen wie folgt interpretiert werden:

Die Kinder der Volksschule lieben das Singen und die Musik und freuen sich, wenn dies in der Schule altersgemäß, fachkompetent und gut unterrichtet wird. Die Studie hat darüber hinaus gezeigt, dass sie auch das zusätzliche musikalische Angebot der Musikschule in hohem Maße schätzen und lieben, wenn es von Musikschulpädagog*innen in Kooperation mit den Klassenlehrer*innen als gemeinsames Singen angeboten wird.

\subsection{Zusammenschau der Ergebnisse aus den Expert*inneninterviews}

Beide befragten Experten der Musikschule der Stadt Innsbruck sowie die Expertinnen der beiden Volksschulen zeigen sich durchwegs begeistert vom Projekt „Singende Schule“.

Bei der Einführung des Projekts gab es keine nennenswerten Schwierigkeiten. Der Unterricht an den Volksschulen durch die Musikpädagogin stellt aus dem Blickwinkel der Direktorinnen und der Lehrerinnen aus musikalischer Sicht für die Schüler*innen eine große Bereicherung dar. Im Besonderen werden den Volksschüler*innen durch die Musikschule der Stadt Innsbruck Auftrittsmöglichkeiten außerhalb des Schulgebäudes ermöglicht. Zu nennen ist hier $u$. a. die Teilnahme einer Volksschule an einem Wettbewerb, die durch die Kooperation mit der Musikschule zustande kam. Dieses Projekt hatte in der Folge großen Einfluss auf die Klassengemeinschaft und den nachfolgenden Musikunterricht.

In Bezug auf das Lehrerteam stellt dieses Projekt aus der Sicht der Befragten ebenfalls einen Mehrwert dar. Jedoch muss an dieser Stelle darauf hingewiesen werden, dass dies differenzierter gesehen werden muss. Volksschulpädagog*innen mit hoher musikalischer Fachkompetenz brauchen die zusätzliche Unterstützung nicht unbedingt. Lehrer*innen, deren Kompetenzschwerpunkt und Primärinteresse nicht im Fach Musikerziehung angesiedelt sind, schätzen diese Hilfestellung dagegen ganz besonders.

Die positive Zusammenarbeit wird auch auf Seiten der Musikschule sehr hervorgehoben. Einigkeit herrschte bei allen Beteiligten, die befragt wurden, dahingehend, dass dieses Projekt mit gutem Gewissen anderen Volksschulen weiterempfohlen werden kann. Aufgrund dieser Erfahrungswerte der letzten Jahre kommen alle befragten Experten der Musikschule sowie die Expertinnen der Volksschule zu dem Schluss, dass die Erhöhung des Intervalls und der daraus resultierende zusätzliche Bedarf an Stunden als Verbesserung angedacht werden sollten. 


\section{Resümee}

Nach Auswertung aller Daten kann von einer Win-Win-Situation für alle Beteiligten gesprochen werden. Von den Kindern der Volksschule wird das Projekt „Singende Schule“ grundsätzlich als sehr positiv bewertet und es wird eine Weiterführung des Projekts befürwortet.

Aus dem Blickwinkel der Direktor*innen stellt dieses Projekt eine Bereicherung für den Musikunterricht dar. Die Leitungen der Volksschulen heben beispielsweise die bessere Ausbildung der Musikpädagog*innen hervor, die einen kompetenteren Zugang zum Fach Musikerziehung ermöglicht. Jedoch zeigten auch Beobachtungen in der Praxis, dass die Volksschulpädagog*innen im Gegenzug meist besser im Umgang mit der Größe bzw. Heterogenität einer Volksschulklasse umgehen können. Für die meisten Volksschulpädagog*innen sei laut Aussage einer Direktorin die Zusammenarbeit eine hervorragende Gelegenheit, Inspirationen, Vorschläge und Ideen für ihren eigenen Musikunterricht zu bekommen. Auch der Stellenwert des Singens an sich wird nach Meinung der Direktor*innen durch diese Art des Unterrichts gesteigert. Ein weiterer positiver Aspekt wird den öffentlichen Auftritten, die außerhalb des Schulgebäudes stattfinden, zugesprochen.

Die befragten Volksschulpädagoginnen sehen die Unterstützung durch die Expertin der Musikschule sehr positiv. Hier wird ebenfalls in erster Linie die intensivere Ausbildung der Musikpädagogin genannt, zumal nach eigener Einschätzung der Pädagog*innen diesbezüglich doch große Unterschiede in der fachlichen Qualifikation bestehen. Die Zusammenarbeit zwischen den Volksschulpädagoginnen und der Musikschulpädagogin an diesen beiden Volksschulen wird sowohl von der Schulleitung als auch von den befragten Lehrerinnen ausnahmslos als „sehr gut“ bezeichnet.

Beleuchtet man das Projekt „Singende Schule“ vonseiten der Musikschule, so ist auch hier ein Mehrwert festzustellen. Die Singschullehrer*innen bekommen durch den Unterricht in der Volksschule die Möglichkeit, gruppendynamische Prozesse kennenzulernen und in ihr eigenes Unterrichtskonzept einzubeziehen. Gerade im Fach Musikerziehung stellt das gegenseitige „Sich-Ansteckenlassen“, ein „Aufeinanderreagieren“, (vgl. Mohr, 1998, S. 52) eine große Bereicherung für die Lehrperson dar.

Vorteilhaft sind auch der Unterricht am Vormittag und die Organisation der Klasseneinteilung innerhalb der Volksschulen. Gerade aus Sicht der Musikschulpädagog*innen wird das als sehr erleichternd angesehen, zumal die Einteilung der Schüler*innen in der Musikschule eine große Herausforderung darstellt (vermehrter Nachmittagsunterricht in der Pflichtschule, intensiv wachsende Freizeitgestaltungen der Schüler*innen).

Darüber hinaus gibt diese Form der Zusammenarbeit der Musikschule die Gelegenheit, auch auf Kinder zuzugehen, die ansonsten eher nicht mit der Musikschule in Berührung kommen würden. Im Rahmen des Unterrichts werden unter anderem Einheiten zur Instrumentenvorstellung abgehalten. Die Erfahrung, die die Kinder in diesen Musikstunden machen, führt immer wieder dazu, dass das ein oder andere Kind ein Musikinstrument erlernen will.

\section{Ausblick und Möglichkeiten der Weiterentwicklung}

Nach Abschluss der Untersuchung zeigt die Auswertung folgende Entwicklungspotenziale:

Es besteht von allen am Projekt beteiligten Personen der einheitliche Wunsch, das Intervall der Betreuung zu erhöhen. Dies würde eine intensivere musikalische Begleitung durch die Musikschulpädagog*innen bedeuten und vermutlich auch den Lernerfolg steigern.

Beide Experten der Musikschule sprechen sich für eine flächendeckende Einführung der Singschule im Gesamtraum der Stadt Innsbruck aus. Die Umsetzung der Intervallerhöhung sowie die flächendeckende Einführung können nur mithilfe der Stadt Innsbruck durch Bereitstellung zusätzlicher Ressourcen erfolgen. Eine Art Rotation des Projekts „Singende Schule“ könnte anderen Innsbrucker Volksschulen ebenfalls die Möglichkeit geben, daran teilnehmen zu können. 
Derzeit existiert für die "Singende Schule“ kein eigener Lehrplan. Der Lehrplan wurde ohne Abänderungen aus der früheren „Singschule“ übernommen. Dieser enthält jedoch ein sehr weites Spektrum der Musikerziehung. Die Umsetzung dieser Vorgaben ist im Zuge dieses Projekts nicht möglich, weil die „Singende Schule“ gegenüber der früheren „Singschule“ einen Bruchteil der Unterrichtsstunden zur Verfügung hat. Es sollte daher in naher Zukunft ein Lehrplan für die "Singende Schule“ von Seiten der Musikschule der Stadt Innsbruck unter Einbezug von Expert*innen aus dem tertiären Bildungsbereich (Musikuniversität, Pädagogische Hochschule) erarbeitet werden.

Ein zentraler Punkt für die Optimierung des Projekts „Singende Schule“ ist der Einsatz von stimmlich und pädagogisch bestens ausgebildeten Musikpädagog*innen.

Abschließend kann festgehalten werden, dass die Ergebnisse der Untersuchung dem Projekt insgesamt ein sehr positives Zeugnis in Bezug auf zusätzlichen Lernerfolg der Schüler*innen sowie den Kompetenzerwerb der Lehrer*innen im Bereich Musikerziehung ausstellen. Die Auswertung hat aber auch einige Optimierungsvorschläge für die Zukunft aufgezeigt, die jedenfalls angedacht und berücksichtigt werden sollten.

\section{Literatur}

AGMÖ. BM:UKK (Hrsg.) (2013). Kompetenzen in Musik. Ein aufbauendes musikpädagogisches Konzept von der Volksschule bis zur kompetenzorientierten Reife- und Diplomprüfung. In: Musikerziehung Spezial, Jahrgang 66, Heft 3, 2013.

Auhagen, W., Bullerjahn, C. \& Höge, H. (Hrsg.). (2007). Musikpsychologie - Musikalische Sozialisation im Kinder- und Jugendalter. Jahrbuch der Deutschen Gesellschaft für Musikpsychologie. Hogrefe Verlag GmbH \& Co. KG.

Bundesministerium für Unterricht, Kunst und Kultur. (2013). Kooperationen von Schulen und Musikschulen. Wien: (Hrsg.) BMUKK. Steuerungsgruppe Musik \& Bildung.

Brosius, F. (2004). SPSS 12. Das wissenschaftliche Standardwerk. Bonn. mitp-Verlag.

Diekmann, A. (2010). Empirische Sozialforschung. Grundlagen, Methoden, Anwendungen. Reinbek bei Hamburg. Rowohlt Verlag. GmbH.

Kurtz, B. (2018). Die Stimme im Lehrberuf. Über die besonderen Anforderungen im pädagogischen Alltag und die Möglichkeiten der Stimmpädagogik im Lehramtsstudium. VOX HUMANA. Fachzeitschrift für Gesangspädagogik. (06/2018) [Hrsg.: Bundesverband deutscher Gesangspädagogen und EVTA-Austria Bund Österreichischer Gesangspädagogen]

Leitfaden der Musikschule der Stadt Innsbruck. (2016). Singende Schule. Innsbruck.

Mohr, A. (1998). Handbuch der Kinderstimmbildung. 2. Auflage. Mainz. Schott.

Pädagogische Hochschule Tirol. (2021). Curriculum. Zugriff am 1.7.2021 unter http://ph-tirol.ac.at/node/90

Stadtarchiv/Stadtmuseum Innsbruck (2018). 200 Jahre Musikverein, Musikschule, Konservatorium in Innsbruck. o.O.

Tiroler Landeskonservatorium. (2021). IGP BA. Zugriff am 1.7.2021 unter https://www.konstirol.at/studienund-curricula/igp-ba/

Wolf, W. (2018). Lehrplan der Volksschule. BGBI. Nr. 134/1963 in der Fassung BGBI. II Nr. 261/2015 mit Anmerkung und Ergänzungen. Graz: Leykam. 